\title{
COLECCIÓN TOMÁS G. ZOEBISCH ASOCIADA AL CENTRO DE ESTUDIOS EN ZOOLOGÍA, UNIVERSIDAD DE GUADALAJARA
}

\author{
Ana Laura González-Hernández y José Luis Navarrete-Heredia* \\ Laboratorio de Entomología, Centro de Estudios en Zoología, CUCBA, Universidad de Guadalajara. \\ Apdo. postal 234, CP 45150, Zapopan, Jalisco, México. \\ *autor para correspondencia:<glenusmx@gmail.com>,<snavarre@cucba.udg.mx>
}

González-Hernández A. L. \& J. L. Navarrete-Heredia. 2011. Colección Tomás G. Zoebisch asociada al Centro de Estudios en Zoología, Universidad de Guadalajara. Acta Zoológica Mexicana (n. s.), 27(2): 463-483.

RESUMEN. En este trabajo se da a conocer información de la colección entomológica Tomás G. Zoebisch, la cual fue donada e incorporada a la colección Entomológica del Centro de Estudios en Zoología, Universidad de Guadalajara. La colección Zoebisch está integrada por 3,790 ejemplares, de los cuales, 838 proceden del extranjero y 2,952 de México. Éstos pertenecen a 31 familias y 436 especies. Las familias mejor representadas son Scarabaeidae (2,601 especímenes), seguida por Carabidae (684). Por su parte Pleocomidae y Ochodaeidae están representadas por un ejemplar. El mayor número de especímenes de México procede del estado de Morelos (697). De los ejemplares extranjeros predominan aquellos de Perú, España y Estados Unidos. La información de los especímenes se encuentra en dos bases de datos: una para ejemplares extranjeros y otra para mexicanos. Copias de las mismas están depositadas en la Comisión Nacional para el Conocimiento y Uso de la Biodiversidad y en el Centro de Estudios en Zoología.

Palabras clave: Colección entomológica, Coleoptera, Centro de Estudios en Zoología.

González-Hernández A. L. \& J. L. Navarrete-Heredia. 2011. The Tomás G. Zoebisch collection asociated to Centro de Estudios en Zoología, University of Guadalajara. Acta Zoológica Mexicana (n. s.), 27(2): 463-483.

ABSTRACT. We provide an overview of the Entomological Collection Tomas G. Zoebisch, recently donated to the Entomological Collection of the Centro de Estudios en Zoologia, Universidad de Guadalajara. This collection contains 3,790 specimens: 2,952 from Mexico and 838 from other countries. They belong to 31 families and 436 species. Scarabaeidae is the most abundant family (2,601 specimens), followed by Carabidae (684), whereas Pleocomidae and Ochodaeidae are represented by only one specimen each one. Most of the specimens from Mexico were collected in Morelos State. Beetles from other countries are well represented from Peru, Spain and, the United States of America. Labels information was included in two databases: one for Mexican beetles and another one for those of other countries.

Recibido: 18/08/2010; aceptado: 14/12/2010. 
Copies of these databases are deposited at the Comision Nacional para el Conocimiento y Uso de la Biodiversidad and in the Centro de Estudios en Zoologia.

Key words: Entomological collection, Coleoptera, Centro de Estudios en Zoologia.

\section{INTRODUCCIÓN}

El orden Coleoptera ocupa el primer lugar como grupo megadiverso. A nivel mundial se conocen cerca de 360,000 especies (Bouchard et al. 2009). Aunque no existe un inventario reciente sobre la diversidad alfa del grupo en México, la estimación más reciente, sugiere la presencia de 20,030 especies (Llorente-Bousquets \& Ocegueda 2008). Ante esta diversidad y el número reducido de especialistas, el trabajo taxonómico del orden en México, se ha concentrado en pocas familias, evidenciando la necesidad de formar especialistas para muchos grupos (Navarrete-Heredia \& Fierros-López 2001). En este contexto, las colecciones entomológicas, juegan un papel primordial al ser reservorios de especímenes que sustentan estudios taxonómicos y sistemáticos.

En el país existen más de 180 colecciones biológicas, de las cuales, una veintena contienen especímenes de Coleoptera (Ordóñez-Reséndiz 2005). La actual colección entomológica del Centro de Estudios en Zoología de la Universidad de Guadalajara (CZUG), se formalizó en 1995 con el objetivo principal de conocer la entomofauna de la región y desarrollar trabajos taxonómicos y sistemáticos con coleópteros asociados a hongos y los pertenecientes a la familia Staphylinidae. Con el tiempo, los grupos estudiados se han incrementado y ahora el trabajo taxonómico se enfoca hacia diferentes grupos de Coleoptera y de Hymenoptera Apoidea y Formicidae.

La colección CZUG está constituida por ejemplares montados en alfiler entomológico, además de muchas muestras en alcohol. Aunque se tienen ejemplares de diferentes órdenes (Odonata, Orthoptera, Blattodea, Isoptera, Hemiptera, Megaloptera, Diptera y Lepidoptera, entre otros), existe un predominio de especímenes de Coleoptera e Hymenoptera. Actualmente se dispone de representantes de más de 1,500 especies provenientes de diferentes localidades de México, además de otros países, obtenidos por intercambio con diversas instituciones de investigación. Detalles sobre la representación de Hemiptera Membracoidea, Megaloptera y Formicidae en la colección CZUG, se pueden consultar en Navarrete-Heredia \& Guerrero-Vázquez (2005).

En diciembre del 2004, Melissa Zoebisch Olvera, hija del Dr. Tomás G. Zoebisch donó la colección de su padre a la Colección Entomológica del Centro de Estudios en Zoología. El Dr. Zoebisch, biólogo egresado de la Universidad Autónoma Metropolitana-Iztapalapa en México; obtuvo los grados de maestría y doctorado en entomología por la Universidad de Florida, Gainesville, Estados Unidos de América. Participó como investigador de control de plagas de insectos utilizando parasitoides, depredadores y entomopatógenos en el Centro Agronómico Tropical de Investigación y 
Enseñanza (CATIE) en Turrialba, Costa Rica. Publicó varios artículos científicos, participó como asesor de varias tesis y trabajos de investigación, formó su colección con ejemplares provenientes de diferentes países por donaciones de colegas y sus propias colectas, realizadas desde su infancia.

En este trabajo y como parte de las actividades de sistematización de la información de la colección entomológica del Centro de Estudios en Zoología, se proporciona el inventario de la Colección Tomás G. Zoebisch (TGZC).

\section{MATERIALES Y MÉTODOS}

Los especímenes se encuentran depositados en cajas entomológicas tipo Cornell y almacenadas en un anaquel metálico. La información de los ejemplares se capturó en dos bases de datos: los ejemplares mexicanos en la base de datos Biotica 4.3 y los ejemplares extranjeros en una base de datos elaborada en Access y que corresponde al modelo mínimo relacional SNIB-2004-2005 diseñado por la Comisión Nacional para el Conocimiento y Uso de la Biodiversidad (CONABIO). Copias de las mismas se encuentran en la CONABIO y en el Centro de Estudios en Zoología.

Previo a la incorporación al espacio de la colección entomológica CZUG, el material se fumigó para prevenir una infestación con derméstidos. Algunos ejemplares se restauraron utilizando goma entomológica para pegar las partes desprendidas. En la mayoría de los casos se mantuvieron las etiquetas originales. Las etiquetas en mal estado o con riesgo de dañar la información fueron sustituidas por etiquetas nuevas. En cada uno de los especímenes (equivalente a un registro en las bases de datos) se consideró la información de colecta y se le agregó un número de catálogo, considerando las siglas de la colección y un número consecutivo (por ejemplo TGZC 0001). Los ejemplares se organizaron de acuerdo con la clasificación de Lawrence \& Newton (1995). Para la nomenclatura de las especies de los diferentes grupos, se utilizaron las propuestas de Lucanidae (Maes 1992), Passalidae (Reyes-Castillo 1970), Geotrupidae (Howden 2003) y Scarabaeidae (Morón et al. 1997, Morón 2003, Sakai \& Nagai 1998). Para la autoridad de géneros y especies se consultó Allard (1985, 1986, 1991), Arce-Pérez \& Morón (2000), Bates (1886-1890), Blackwelder (1944), Cazier (1954), Chemsak et al. (1992), Dellacasa et al. (2001, 2002), Deuve (2004), Hansen (1999), Hardy (1975), Hawks (2001), Howden y Cartwright (1963), Howden (1964, 2003), Maes (1992), Krajč́́k (1998, 1999), Lachaume (1983), Mico y Galante (2002), Morón (1993), Nilsson (2001), Ratcliffe (1976, 1991), Ratcliffe \& Cave (2006), Solís (2007), Smith (2003), Trauther \& Geigenmüller (1987), Woodruff (1973), Woodruff \& Beck (1989), Young (1988). Para cada familia, las especies se encuentran ordenadas alfabéticamente. Durante este proceso, parte del material de Scarabaeoidea, fue determinado por el Dr. Pedro Reyes-Castillo, Dr. Cuauhtémoc Deloya y M. C. Leonardo Delgado del Instituto de Ecología, A.C., además de personal del CZUG. 


\section{RESULTADOS Y DISCUSIÓN}

La colección Zoebisch se incorporó a la colección entomológica del Centro de Estudios en Zoología de la Universidad de Guadalajara en una sección independiente. Está representada por 3,790 ejemplares de los cuales 838 proceden del extranjero y 2,952 de México. Estos ejemplares están incluidos en 30 familias y 436 especies (Apéndice 1). La familia con mayor número de ejemplares es Scarabaeidae con 2,061, seguida de Carabidae con 648, mientras que las familias Chrysomelidae, Cerambycidae, Passalidae, Geotrupidae y Lucanidae tienen entre 50 y 130 ejemplares. Algunas familias están representadas por menos de treinta ejemplares (Curculionidae, Nitidulidae, entre otras) y finalmente, Trogossitidae, Ochodaeidae y Pleocomidae, tienen dos (la primera) o un ejemplar, las dos restantes (Cuadro 1).

Los ejemplares extranjeros proceden principalmente de Perú (245), España (200), Estados Unidos de América (176), aunque en total se tienen representantes de 38 países (Cuadro 2). Es importante señalar la existencia de dos paratipos procedentes de Brasil: Agaocephala margaridae Alvarenga, 1958, únicos ejemplares que se incorporaron a la sección de ejemplares tipo de la colección CZUG. Entre los 2,952 ejemplares mexicanos, una cantidad importante procede del estado de Morelos (697), Veracruz (460), Jalisco (361), Estado de México (323), Puebla (228) y Distrito Federal (226). Así mismo, Morelos es el estado mejor representado en cuanto al número de especies (74), seguida de Veracruz (72), Puebla (48), Estado de México (43), Jalisco (40), Chiapas (31), Michoacán (24), Distrito Federal (21), Guerrero (17), Hidalgo (10) y el resto de los estados están representados por menos de diez especies (Cuadro 3).

Entre las especies con mayor número de ejemplares se encuentran: Neoscelis dohrni con 64 ejemplares, Cotinis mutabilis (57), Onthophagus batesi (36), Attavicinus monstrosus, Euphoria basalis, Strategus aloeus y Tomarus sallei con 30 cada una, Strigoderma sulcipennis (29), Xyloryctes howdenorum (27), Euphoria biguttata (25), Euphoria pulchella (24), Euphoria subtomentosa y Megasoma occidentalis con 22 cada una y Anomala cincta, Canthon cyanellus y Dynastes hyllus con 21 especímenes cada especie.

\section{COMENTARIOS FINALES}

Como parte del trabajo taxonómico que desarrolla el personal de la Colección Entomológica del Centro de Estudios en Zoología, la incorporación del material entomológico de la Colección Zoebisch incrementa de manera importante el acervo de la colección tanto en número de especies como en número de individuos. Actualmente, la base de datos de la colección cuenta con 13,433 ejemplares y 359 especies exclusivamente de Coleoptera, que sumados a los 3,790 ejemplares y 436 especies de la Colección Zoebisch, da un total de 17,223 especímenes representantes de más de 
Cuadro 1. Número de especímenes por familia de la colección Tomas G. Zoebisch.

\begin{tabular}{|c|c|c|c|c|c|}
\hline Familias & $\begin{array}{l}\text { Ejemplares } \\
\text { mexicanos }\end{array}$ & $\begin{array}{l}\text { Ejemplares } \\
\text { extranjeros }\end{array}$ & $\begin{array}{l}\text { Número de } \\
\text { especies }\end{array}$ & $\begin{array}{c}\text { Ejemplares sin } \\
\text { datos }\end{array}$ & $\begin{array}{c}\text { Total de } \\
\text { ejemplares }\end{array}$ \\
\hline Dytiscidae & 7 & 5 & 1 & - & 12 \\
\hline Carabidae & 547 & 130 & 66 & 7 & 684 \\
\hline Hydrophilidae & 1 & 1 & - & - & 2 \\
\hline Histeridae & 4 & 5 & - & - & 9 \\
\hline Silphidae & 2 & 5 & 4 & - & 7 \\
\hline Staphylinidae & 3 & 3 & 2 & - & 6 \\
\hline Lucanidae & - & 49 & 16 & - & 49 \\
\hline Passalidae & 66 & 15 & 19 & 2 & 83 \\
\hline Trogidae & 15 & 1 & 4 & - & 16 \\
\hline Pleocomidae & - & 1 & 1 & - & 1 \\
\hline Geotrupidae & 10 & 47 & 15 & - & 57 \\
\hline Ochodaeidae & - & - & - & 1 & 1 \\
\hline Hybosoridae & 10 & 2 & 1 & - & 12 \\
\hline Scarabaeidae & 2140 & 415 & 302 & 46 & 2601 \\
\hline Buprestidae & 3 & 1 & - & - & 4 \\
\hline Lycidae & 1 & 4 & - & - & 5 \\
\hline Lampyridae & 4 & 16 & - & - & 20 \\
\hline Cantharidae & 14 & - & - & - & 14 \\
\hline Dermestidae & - & 5 & - & - & 5 \\
\hline Trogossitidae & - & 2 & - & - & 2 \\
\hline Cleridae & - & 3 & 1 & - & 3 \\
\hline Melyridae & - & 4 & - & - & 4 \\
\hline Nitidulidae & 5 & - & - & - & 5 \\
\hline Erotylidae & 2 & 18 & - & - & 20 \\
\hline Coccinellidae & 14 & 2 & - & - & 16 \\
\hline Tenebrionidae & 10 & 14 & 1 & - & 24 \\
\hline Oedemeridae & - & 1 & 1 & - & 1 \\
\hline Cerambycidae & 65 & 19 & 6 & 3 & 87 \\
\hline Chrysomelidae & 66 & 66 & 6 & 1 & 133 \\
\hline Brentidae & 2 & 5 & - & - & 7 \\
\hline Curculionidae & 18 & 11 & 2 & - & 29 \\
\hline
\end{tabular}


Cuadro 2. Número de especímenes y especies del extranjero representadas en la colección Tomás G. Zoebisch. ND. Sin datos.

\begin{tabular}{|c|c|c|}
\hline Continente o país & Número de ejemplares & Número de especies \\
\hline África & 2 & 2 \\
\hline Alemania & 24 & 10 \\
\hline Argentina & 5 & 3 \\
\hline Australia & 1 & 1 \\
\hline Brasil & 9 & 6 \\
\hline Camerún & 6 & 2 \\
\hline Canadá & 1 & 1 \\
\hline Chile & 2 & 1 \\
\hline Colombia & 6 & 2 \\
\hline Costa de Marfil & 1 & 1 \\
\hline Costa Rica & 4 & 1 \\
\hline Ecuador & 1 & 1 \\
\hline España & 200 & 63 \\
\hline Estados Unidos de América & 176 & 35 \\
\hline Filipinas & 2 & 1 \\
\hline Francia & 33 & 11 \\
\hline Gabón & 1 & 1 \\
\hline Ghana & 4 & 2 \\
\hline India & 5 & 1 \\
\hline Indonesia & 1 & 1 \\
\hline Italia & 3 & 3 \\
\hline Japón & 28 & 9 \\
\hline Madagascar & 2 & 1 \\
\hline Malasia & 1 & 1 \\
\hline Nambia & 1 & 1 \\
\hline Nueva Guinea & 5 & 2 \\
\hline ND & 1 & - \\
\hline Perú & 245 & 20 \\
\hline Republica Centroafricana & 17 & 8 \\
\hline Republica Checa & 1 & 1 \\
\hline $\begin{array}{l}\text { Republica Democrática del } \\
\text { Congo }\end{array}$ & 28 & 10 \\
\hline Taiwán & 2 & 1 \\
\hline Tayikistán & 3 & - \\
\hline Trinidad y Tobago & 2 & 1 \\
\hline Túnez & 1 & - \\
\hline Uganda & 2 & 1 \\
\hline Uruguay & 1 & 1 \\
\hline Venezuela & 4 & 1 \\
\hline
\end{tabular}


Cuadro 3. Número de especímenes y especies mexicanas representadas en la Colección Tomás G. Zoebisch.

\begin{tabular}{|c|c|c|}
\hline Estado & Número de ejemplares & Número de especies \\
\hline Baja California & 1 & 1 \\
\hline Chiapas & 72 & 31 \\
\hline Distrito Federal & 226 & 21 \\
\hline Durango & 4 & 2 \\
\hline Estado de México & 323 & 43 \\
\hline Guerrero & 72 & 17 \\
\hline Guanajuato & 17 & 5 \\
\hline Hidalgo & 42 & 10 \\
\hline Jalisco & 361 & 40 \\
\hline Michoacán & 123 & 24 \\
\hline Morelos & 697 & 74 \\
\hline Nuevo León & 4 & 2 \\
\hline Oaxaca & 21 & 9 \\
\hline Puebla & 228 & 48 \\
\hline Quintana Roo & 1 & 1 \\
\hline Querétaro & 33 & 9 \\
\hline San Luis Potosí & 7 & 2 \\
\hline Sinaloa & 1 & 1 \\
\hline Tamaulipas & 4 & 2 \\
\hline Tlaxcala & 4 & 1 \\
\hline Tabasco & 6 & 6 \\
\hline Veracruz & 460 & 72 \\
\hline Yucatán & 18 & 4 \\
\hline
\end{tabular}

700 especies. Con la incorporación de material extranjero, la representatividad taxonómica se incrementa de manera importante, particularmente dentro de las familias Lucanidae y Scarabaeidae (especialmente Cetoniinae).

AGRADECIMIENTOS. A Melissa Zoebisch Olvera por su confianza al donar la colección de su papá al Centro de Estudios en Zoología. A la Comisión Nacional para el Conocimiento y Uso de la Biodiversidad por los recursos económicos otorgados para la realización de este proyecto. A la Universidad de Guadalajara por su constante apoyo al trabajo en la Colección Entomológica a través de los proyectos con recursos de la misma institución. Al Dr. P. Reyes-Castillo, Dr. C. Deloya, M.C. Leonardo Delgado por su apoyo en la determinación de algunos especímenes. 


\section{LITERATURA CITADA}

Allard, V. 1985. Les coleóptères du monde. The beetles of the World. Vol. 6. Goliathini 2. Sciences Nat, Compiègne, France.

Allard, V. 1986. Les coleóptères du monde. The beetles of the World. Vol. 7. Goliathini 3. Sciences Nat, Compiègne, France.

Allard, V. 1991. Les coleóptères du monde. The beetles of the World. Vol. 11. Goliathini 4. Sciences Nat, Compiègne, France.

Arce-Pérez, R. \& M. A. Morón. 2000. Taxonomía y distribución de las especies de Macrodactylus Latreille (Coleoptera: Melolonthidae) en México y Estados Unidos de América. Acta Zoológica Mexicana (n.s.), (79): 181-182.

Bates, H. W. 1886-1890. Biología Centrali-Americana. Insecta. Coleoptera. Vol. II: parte 2. Pecticornia and Lamellicornia. Taylor and Francis, London.

Blackwelder, R. 1944. Checklist of the coleopterous insects of Mexico, Central America the West Indies, and South America. Smithsonian Institution United States National Museum Bulletin, (2): 189-341.

Bouchard, P., V.V. Grebennikov, A.B.T. Smith \& H. Douglas. 2009. Biodiversity of Coleoptera. Pp. 265-301. In: R. Foottit \& P. Adler (Eds.). Insect biodiversity: Science and Society. Blackwell Publishing, West Sussex, UK.

Cazier, M. 1954. A review of the Mexican tiger beetles of the genus Cicindela (Coleoptera, Cicindelidae). Bulletin of the American Museum of Natural History, 103: 227-310.

Chemsak, J. A., E. G. Linsley y F. A. Noguera. 1992. Listados faunísticos de México II. Los Cerambycidae y Disteniidae de Norteamérica, Centroamérica y las Indias Occidentales (Coleoptera). Universidad Nacional Autónoma de México, México, D.F.

Dellacasa, G., P. Bordat and M. Dellacasa. 2001. A revisional essay of the world genus-group taxa of Aphodiinae (Coleoptera Aphodiidae). Memorie della Società Entomologica Italiana, 79: 1-482.

Dellacasa, M., R.D. Gordon, and G. Dellacasa. 2002. Aphodiinae described or recorded by Bates in Biología Centrali-Americana (Coleoptera Scarabaeoidae: Aphodiidae). Acta Zoológica Mexicana (n.s.), (86): 155-223.

Deuve, T. 2004. Illustrated catalogue of the genus Carabus of the World (Coleoptera: Carabidae). Pensoft, Sofia \& Moscow.

Hansen, M. 1999. World catalog of insects. Vol. 2. Hydrophiloidea (Coleoptera). Apollo Books, Stenstrup, Denmark.

Hardy, A. R. 1975. A revision of the genus Pelidnota of America North of Panama Coleoptera: Scarabaeidae; Rutelinae. University of California Publications in Entomology, 78: 1-43.

Hawks, C. D. 2001. Taxonomic and nomenclatural changes in Chrysina and a synonymic checklist of species (Scarabaeidae: Rutelinae). Occasional Papers of the Consortium Coleopterorum, 4: 1-8.

Howden, H. F. 1964. The Geotrupinae of North and Central America. Memoirs of the Entomological Society of Canada, (39): 1-91.

Howden, H. F. 2003. Subfamilia Geotrupinae. Pp. 95-106. En:M.A. Morón (ed.). Atlas de los escarabajos de México. Coleoptera: Lamellicornia. Vol. II. Familias Scarabaeidae, Trogidae, Passalidae y Lucanidae. Argania Editio, Barcelona.

Howden, H. F. \& O. L. Cartwright. 1963. Scarab beetles of the genus Onthophagus Latreille North of Mexico (Coleoptera: Scarabaeidae). Proceedings of the United States National Museum, 114: $1-135$.

Krajčík, M. 1998. Cetoniidae of the World catalogue-Part I. (Coleoptera: Cetoniidae). Typos Studio Most, Czech Republic. 
Krajčík, M. 1999. Cetoniidae of the World catalogue- Part II. (Coleoptera: Cetoniidae). Typos Studio Most, Czech Republic.

Lachaume, G. 1983. Les coleóptères du monde. The beetles of the World. Vol. 11. Goliathini 3. Sciences Nat, Compiègne, France.

Lawrence, J.F. and A.F. Newton, Jr. 1995. Families and subfamilies of Coleoptera (with selected genera, notes, references and data on family group names). Pp. 779-1006. In: J. Pakaluk and S.A. Ślipiński (Eds.) Biology, Phylogeny, and Classification of Coleoptera. Papers celebrating the $80^{\text {th }}$ Birthday of Roy A. Crowson. Muzeum i Instytut Zoologii PAN, Warszawa.

Llorente-Bousquets, J. \& S. Ocegueda. 2008. Estado del Conocimiento de la biota. Pp. 283-322. En: Capital Natural de México. Vol. I. Conocimiento actual de la biodiversidad. CONABIO, México, D.F.

Maes, J-M. 1992. Lista de los Lucanidae (Coleoptera) del mundo. Revista Nicaragüense de Entomología, 22: 1-121.

Mico, E. \& E. Galante. 2002. Atlas fotográfico de los escarabajos florícolas Ibero-Baleares. Argania Editio, Barcelona.

Morón, M. A. 1993. Catálogo de tipos depositados en la colección M. A. Morón, Xalapa, Veracruz, México (M-XAL) (Insecta, Coleoptera: Lamellicornia). Publicación especial de la Sociedad Mexicana de Entomología, A. C. y Sociedad Veracruzana de Zoología A. C., Xalapa, Veracruz.

Morón, M. A. 2003. Familia Scarabaeidae. Pp. 19-74. En: M.A. Morón (ed.). Atlas de los escarabajos de México. Coleoptera: Lamellicornia. Vol. II. Familias Scarabaeidae, Trogidae, Passalidae y Lucanidae. Argania Editio, Barcelona.

Morón, M. A., B. C. Ratcliffe \& C. Deloya. 1997. Atlas de los escarabajos de México. Coleoptera: Lamellicornia. Vol. I. Familia Melolonthidae. Comisión Nacional para el uso y Conocimiento de la Biodiversidad, Sociedad Mexicana de Entomología, México, D.F.

Navarrete-Heredia, J. L. \& H. E. Fierros-López. 2001. Coleoptera de México: Situación actual y perspectivas de estudio. Pp. 1-21. In: J. L., Navarrete-Heredia, H. E. Fierros-López y A. BurgosSolorio (Eds). Tópicos sobre Coleoptera de México. Universidad de Guadalajara, Universidad Autónoma del estado de Morelos, Guadalajara, Jalisco.

Navarrete-Heredia J. L. \& S. Guerrero-Vázquez (Eds). 2005. Colecciones Zoológicas del Centro de Estudios en Zoología. Universidad de Guadalajara, Guadalajara, Jalisco.

Nilsson, A. 2001. World catalog of Insects. Vol. 3. Dytiscidae (Coleoptera). Apollo Books, Stenstrup, Denmark.

Ordóñez-Reséndiz, M. M. 2005. Colección de Coleoptera (Insecta) de la Facultad de Estudios Superiores de Zaragoza, UNAM. Acta Zoológica Mexicana (n. s.), 21: 96-97.

Ratcliffe, B. C. 1976. A revision of the genus Strategus (Coleoptera: Scarabaeidae). Bulletin of the University of Nebraska State Museum, 10: 93-204.

Ratcliffe, B. C. 1991. The scarab beetles of Nebraska. Bulletin of the University of Nebraska State Museum, 12: 1-333.

Ratcliffe, B.C. \& R. D. Cave. 2006. The Dynastinae scarab beetles of Honduras, Nicaragua and El Salvador (Coleoptera: Scarabaeidae: Dynastinae). Bulletin of the University of Nebraska State Museum, 21: 1-424.

Reyes-Castillo, P. 1970. Coleoptera, Passalidae: Morfología y división en grandes grupos; géneros americanos. Folia Entomologica Mexicana (20-22): 1-240.

Sakai, K. \& S. Nagai. 1998. The Cetoniine beetles of the World. Mushi-Sha, Tokyo.

Solís, A. 2007. La superfamilia Scarabaeoidea de Costa Rica. http://www.inbio.ac.cr/papers/lameli/index.html. Consultado: 24 de mayo 2007. 
Smith, A. B. T. 2003. Checklist of the Scarabaeoidea of the Nearctic realm: including Canada, the continental United States, and the northern Mexican states of Baja California, Baja California Sur, Chihuahua, Coahuila de Zaragoza, Durango, Nuevo Leon, Sinaloa, Sonora, Tamaulipas, and Zacatecas.

http://www-museum.unl.edu/research/entomology/Nearctic-scarabs3.pdf. Consultado: 16 de mayo 2006.

Trauther, J. \& K. Geigenmüller. 1987. Tiger Beetles ground beetles: Illustrated key to the Cicindelidae and Carabidae of Europe. Josef Margraf Publisher, Stuttgart.

Woodruff, R. 1973. The scarab beetles of Florida (Coleoptera: Scarabaeidae) part I. The Laparosticti (Subfamilies: Scarabaeinae, Aphodiinae, Hybosorinae, Ochodaeinae, Geotrupinae, Acanthocerinae). Arthropods of Florida and Neighboring Land Areas, 8: 1-220.

Woodruff, R. \& B. M. Beck. 1989. The scarab beetles of Florida (Coleoptera: Scarabaeidae). Part II. The May or June beetles (genus Phyllophaga). Arthropods of Florida and Neighboring Land Areas, 13: $1-226$.

Young, M. R. 1988. A monograph of the Genus Polyphylla Harris in America north of Mexico (Coleoptera. Scarabaeidae: Melolonthinae). Bulletin of the University of Nebraska State Museum, 11: 1-106.

\section{APÉNDICE 1}

Lista de especies de la Colección Entomológica Tomás G. Zoebisch depositada en la Colección del Centro de Estudios en Zoología, Universidad de Guadalajara (CZUG). El arreglo de subórdenes y familias se basa en Lawrence y Newton (1995). La lista de especies para cada familia está en orden alfabético.

Coleoptera

Adephaga

Dytiscidae Leach, 1815

Meladema coriacea Laporte, 1835

Carabidae Latreille, 1802

Abax parallelepipedus (Piller \& Mitterpacher, 1783)

Agonum sp.

Agonum scutiferum Bates, 1878

Amara sp.

Amara californica Dejean, 1828

Anchomenus dorsalis (Pontoppidan, 1763)

Anthia sp.

Anisotarsus sp.

Apristus mexicanus Bates, 1883

Aptinus displossor (Dufour, 1811)

Bembidion sp.

Brachinus mexicanus Dejean, 1831

Brachinus sclopeta (Fabricius, 1792)

Calatus sp.

Calathus leechi Ball \& Négre, 1972

Calosoma angulatum Chevrolat, 1834

Calosoma atroviren obscurum Géhin, 1885 
Calosoma laeve Dejean, 1826

Calosoma peregrinator Guérin-Méneville, 1844

Calosoma protractum LeConte, 1852

Calosoma retusum Fabricius, 1792

Cassolaia maura (Linnaeus, 1758)

Carabus sp.

Carabus auratus Linnaeus, 1761

Carabus auronitens Fabricius, 1792

Carabus catenulatus Scopili, 1763

Carabus coriaceus Linnaeus, 1758

Carabus fairmairei Thomson, 1875

Carabus granulatus Linnaeus, 1758

Carabus intricatus Linnaeus, 1761

Carabus melancholicus Fabricius, 1798

Carabus monilis Fabricius, 1792

Carabus splendens Olivier, 1790

Chlaenius sp.

Chlaenius chrysocephalus (Rossi, 1790)

Chlaenius festivus (Panzer, 1796)

Chlaenius vestitas (Paykull, 1790)

Cicindela sp.

Cicindela aeneicollis Bates, 1881

Cicindela aterrima Klug, 1834

Cicindela campestri Linnaeus, 1758

Cicindela curvata Chevrolat, 1834

Cicindela flohri Bates, 1879

Cicindela leuconoe Bates, 1890

Cicindela rufiventris sedecimpunctata Klug, 1834

Cicindela sylvicola (Dejean, 1822)

Cicindela sommeri Mannerheim, 1837

Colpodes sp.

Colpodes coerulea Chaudoir, 1859

Cylindera paludosa (Dufour, 1820)

Cymindis axillaris (Fabricius, 1794)

Diachromus germanus (Linnaeus, 1758)

Euchroa sp.

Galerita mexicana Chaudoir, 1872

Galerita strandi Liebke, 1939

Harpalus liobasis Chaudoir, 1868

Licinus punctatulus Fabrisius, 1792

Lophyra flexuosa (Fabricius, 1787)

Megacephala sobrina Dejean, 1831

Morion lafertei Guérin-Méneville, 1844

Notiobia sp.

Notiobia mexicana Dejean, 1829

Nebria brevicollis (Fabricius, 1792)

Onypterygia fulgens Dejean, 1831 
Panagaeus sallei Chaudoir, 1861

Pasimachus californicus Chaudoir, 1850

Pasimachus metallicus Chaudoir, 1880

Pasimachus subangulatus Chaudoir, 1862

Percus patruelis (Dufour, 1820)

Pinacodera sp.

Platynus monachus Dejean, 1831

Poecilus cupreus (Linnaeus, 1758)

Polpochila sp.

Pterostichus melanarius (Illiger, 1798)

Pterostichus mexicanus Chaudoir, 1876

Selenophorus sp.

Selenophorus aequinoctialis Dejean, 1829

Stenomorphus sp.

Stenomorphus californicus Ménétriés, 1844

Stenomorphus penicillatus Darlington, 1936

Zabrus angustatus Rambur, 1838

Polyphaga

Hydrophilidae Latreille, 1802

Histeridae Gyllenhal, 1808

Silphidae Latreille, 1807

Nicrophorus humator (Gleditsch, 1767)

Nicrophorus mexicanus Matthews, 1888

Nicrophorus vespillo (Linnaeus, 1758)

Thanatophilus rugosus (Linnaeus, 1758)

Staphylinidae Latreille, 1802

Creophilus maxillosus (Linnaeus, 1758)

Platydracus fulvomaculatus (Nordmann, 1837)

Lucanidae Latreille, 1804

Dorcus parallelipipedus (Linnaeus, 1785)

Ceruchus piceus (weber, 1801)

Chiasognathus granti Stephens, 1831

Figulus binodulus Waterhouse, 1873

Homoderus mellyi Parry, 1862

Lamprima adolphinae (Gestro, 1875)

Leptinopterus tibialis (Eschscholtz, 1822)

Lucanus cervus (Linnaeus, 1758)

Lucanus capreolus (Linnaeus, 1763)

Lucanus maculifemoratus Motschulsky, 1861

Odontolabis cuvera Hope, 1842

Prosopocoilus antilopus (Swederus, 1787)

Prosopocoilus downesii (Hope, 1835)

Prosopocoilus inclinatus (Motschulsky, 1857)

Serrognathus arfakianus Lansberge, 1880

Sinodendron cylindricum (Linnaeus, 1758)

Passalidae Leach, 1815

Odontotaenius disjuntus (Illiger, 1800) 
Odontotaenius striatopunctatus (Percheron, 1835)

Odontotaenius zodiacus (Truqui, 1857)

Oileus heros (Truqui, 1857)

Oileus rimator (Truqui, 1857)

Passalus cognatus Truqui, 1857

Passalus inops Truqui, 1857

Passalus interstitialis Eschscholtz, 1829

Passalus interruptus (Linnaeus, 1758)

Passalus punctatostriatus Percheron, 1835

Passalus punctiger LePeletier \& Serville, 1825

Passalus rhodocanthopoides (Kuwert. 1891)

Paxillus leachi MacLeay, 1819

Proculus goryi (Melly, 1833)

Proculus opacipennis (Thomson, 1857)

Pseudacanthus mexicanus (Truqui, 1857)

Ptichopus angulatus (Percheron, 1835)

Verres cavicollis Bates, 1886

Verres corticicola (Truqui, 1857)

Trogidae MaCleay, 1819

Omorgus punctatus (Germar, 1824)

Omorgus suberosus (Fabricius, 1775)

Omorgus tessellatus LeConte, 1854

Trox plicatus Robinson, 1940

Pleocomidae LeConte, 1861

Pleocoma australis Fall, 1911

Geotrupidae Latreille, 1802

Ceratotrupes bolivari Halffter \& Martinez, 1847

Ceratotrupes fronticornis Erichson, 1847

Cnemotrupes splendidus (Fabricius, 1775)

Geohowdenius egeriei Germar, 1824

Geotrupes sp.

Geotrupes guatemalensis Bates, 1887

Geotrupes mutator (Marsham, 1802)

Geotrupes stercoriarus (Linnaeus, 1758)

Megatrupes fisheri Howden, 1967

Mycotrupes gaigei Olson \& Hubbell, 1954

Neoathyreus interruptus Howden, 1964

Neoathyreus mexicanus Klug, 1845

Onthotrupes herbeus Jekel, 1866

Onthotrupes sallei Jekel, 1866

Peltotrupes profundus Howden, 1952

Sericotrupes niger (Marsham, 1802)

Thorectes chersinus Delabie, 1954

Hybosoridae Erichson, 1847

Hybosorus illigeri Reiche, 1853

Scarabaeidae Latreille, 1802

Agaocephala margaridae Alvarenga, 1958 
Aethiessa floralis (Fabricius, 1787)

Amadotrogus vicinus (Mulsant, 1842)

Amithao cavifrons (Burmeister, 1842)

Amithao erythropus (Burmeister, 1842)

Amithao pyrrhonotus (Burmeister, 1842)

Ammoecius elevatus (Olivier, 1789)

Ancognatha quadripunctata Bates, 1888

Anomala sp.

Anomala castaniceps Bates, 1888

Anomala cincta Say, 1835

Anomala donovani Stephens, 1830

Anomala hoepfneri Bates, 1888

Aphodius sp.

Aphodius opisthius Bates, 1887

Archophileurus vervex (Burmeister, 1847)

Argyripa porioni Arnaud, 1988

Aspidolea singularis Bates, 1888

Ataenius sp.

Ataenius cognatus LeConte, 1858

Ataenius complicatus Harold, 1869

Ataenius crenulatus Schmidt, 1910

Ataenius cribrithorax Bates, 1887

Attavicinus monstrosus (Bates, 1887)

Bothynus complanus (Burmeister, 1847)

Callistethus cupricollis Chevrolat, 1834

Calomacraspis concinna (Blanchard, 1850)

Calomacraspis haroldi (Candéze, 1869)

Calomacraspis splendens (Burmeister, 1844)

Canthidium centrale Boucomont, 1928

Canthon sp.

Canthon cyanellus LeConte, 1859

Canthon femoralis (Chevrolat, 1834)

Canthon humectus assimilis Robinson, 1946

Canthon humectus hidalgoensis Bates, 1887

Canthon humectus humectus Say, 1831

Canthon indigaceus chevrolati Harold, 1868

Canthon imitator Brown, 1946

Canthon morsei Howden, 1966

Canthon piluliformis (Blanchard, 1846)

Canthon subhyalinus Harold, 1867

Canthon vazquezae (Martínez, Halffter \& Halffter, 1964)

Cetonia aurata Linnaeus, 1761

Cetonia carthami Gory \& Percheron, 1833

Ceraspis sp.

Ceraspis pilatei (Harold, 1863)

Chalcosoma atlas (Linnaeus, 1758)

Chalcosoma chiron Olivier, 1789 
Charadronota quadrisignata (Gory \& Percheron, 1833)

Chasmodia collaris Blanchard, 1850

Chlorixanthe flavoviridis (Thompson, 1860)

Chlorocala africana (Drury, 1733)

Chlorota limbaticollis Blanchard, 1850

Chrysina sp.

Chrysina adelaida (Hope, 1840)

Chrysina aenigmatica (Morón, 1990)

Chrysina auripes Gray, 1832

Chrysina badeni (Boucard, 1878)

Chrysina beyeri (Skinner, 1905)

Chrysina costata (Blanchard, 1850)

Chrysina diversa (Ohaus, 1912)

Chrysina gloriosa LeConte, 1854

Chrysina laniventris (Sturm, 1843)

Chrysina lecontei (Horn, 1882)

Chrysina macropus (Francillon, 1795)

Chrysina peruviana Kirby, 1828

Chrysina psittacina (Sturm, 1843)

Coelorrhina barthi (Harold, 1878)

Coelorrhina superba Gerstaecker, 1882

Coelosis biloba (Linnaeus, 1767)

Copris armatus Harold, 1869

Copris boucardi Harold, 1869

Copris incertus Say, 1835

Copris klugi Harold, 1869

Copris laeviceps Harold, 1869

Copris lugubris Boheman, 1858

Copris minutus (Drury, 1773)

Copris rebouchei Harold, 1869

Coprimorphus scrutator (Herbst, 1789)

Coprophanaeus pluto (Harold, 1863)

Coprophanaeus telamon corythus Harold, 1863

Cotalpa consobrina (Horn, 1871)

Cotinis laticornis Bates, 1889

Cotinis mutabilis (Gory \& Percheron, 1833)

Cotinis nitida (Linnaeus, 1764)

Cotinis orientalis Deloya \& Ratcliffe, 1988

Cotinis pauperula (Gory \& Percheron, 1833)

Cotinis subviolacea Gory \& Percheron, 1833

Cremastocheilus beameri Cazier, 1940

Cyclidius sp.

Cyclocephala sp.

Cyclocephala amazona (Linnaeus, 1767)

Cyclocephala amblyopsis Bates, 1888

Cyclocephala barrerai Martínez, 1969

Cyclocephala carbonaria Arrow, 1911 
Cyclocephala deceptor (Casey, 1915)

Cyclocephala fasciolata Bates, 1888

Cyclocephala guttata Bates, 1888

Cyclocephala jalapensis Casey, 1915

Cyclocephala lunulata Burmeister, 1847

Cyclocephala lurida Bland, 1863

Cyclocephala mafaffa Burmeister, 1847

Cyclocephala marginicollis Arrow, 1902

Cyclocephala melanocephala (Fabricius, 1775)

Cyclocephala picta Burmeister, 1847

Cyclocephala sanguinicollis Burmeister, 1847

Cyclocephala sexpunctata Laporte, 1840

Cyclocephala sororia Bates, 1888

Cyclocephala stictica Burmeister, 1847

Cyclocephala warneri Ratcliffe, 1992

Cyclocephala weidneri Endrödi, 1964

Cyprolais loricata (Janson, 1877)

Cyprolais selene (Kolbe, 1899)

Deltochilum gibbosum sublaeve Bates, 1887

Deltochilum pseudoparile Paulian, 1938

Deltochilum scabriusculum Bates, 1887

Dichotomius amplicollis Harold, 1869

Dichotomius colonicus (Say, 1835)

Dichotomius satanas (Harold, 1867)

Dicronorhina micans (Drury, 1773)

Digitonthophagus gazella (Fabricius, 1787)

Diplotaxis sp.

Diplotaxis bowditchi Fall, 1909

Diplotaxis cribaticollis Blanchard, 1851

Diplotaxis mediafusca Vaurie, 1960

Diplotaxis simplex Blanchard, 1851

Diplotaxis tarsalis Schaeffer, 1907

Diloboderus abderus Sturm, 1826

Dynastes hyllus Chevrolat, 1843

Dynastes hercules (Linnaeus, 1758)

Dynastes neptunus Quenzel, 1817

Dyscinetus dubius (Olivier, 1789)

Dyscinetus laevipunctatus Bates, 1888

Dyscinetus morator (Fabricius, 1789)

Dyscinetus picipes (Burmeister, 1847)

Enema pan (Fabricius, 1775)

Epectinaspis mexicana (Burmeister, 1844)

Eudicella gralli (Buquet, 1836)

Eudicella smithi (MacLeay, 1838)

Euphoria sp.

Euphoria basalis (Gory \& Percheron, 1833)

Euphoria biguttata (Gory \& Percheron, 1833) 
Euphoria candezei Janson, 1875

Euphoria canescens (Gory \& Percheron, 1833)

Euphoria dimidiata (Gory \& Percheron, 1833)

Euphoria geminata (Chevrolat, 1835)

Euphoria inda (Linnaeus, 1764)

Euohoria kerni (Haldeman, 1852)

Euphoria pulchella (Gory \& Percheron, 1833)

Euphoria sepulcralis leucographa (Gory \& Percheron, 1833)

Euphoria subtomentosa (Dejean, 1837)

Euphoria westermanni(Gory \& Percheron, 1833)

Eurysternus angustulus Harold, 1869

Eurysternus caribaeus (Herbst, 1789)

Eurysternus foedus Guérin, 1844

Eurysternus magnus Castelnau, 1840

Eurysternus mexicanus Harold, 1869

Eurysternus velutinus Bates, 1887

Gametis jucunda (Faldermann, 1835)

Genuchinus digitatus Krikken, 1981

Glycyphana fulvistemma Motschulsky, 1858

Goliatus goliatus (Linnaeus, 1771)

Goliatus orientalis Moser, 1909

Goliatus regius Klug, 1835

Golofa pizarro Hope, 1837

Golofa porteri Hope, 1837

Golofa pusilla Arrow, 1911

Gromphas sp.

Gromphas aeruginosa Perty, 1830

Gromphas lacordairei Brulle, 1834

Gymnetina sp.

Gymnetina cretacea (LeConte, 1866)

Gymnetis bajula Olivier, 1789

Gymnetis bajula radicollis Burmeister, 1847

Gymnetis hebraica Drapiez, 1820

Gymnetis stellata (Latreille, 1833)

Gymnetosoma chontalensis (Janson, 1875)

Gymnetosoma pantherina Burmeister, 1842

Gymnopleurus mopsus Palls, 1781

Hemiphileurus dejeani (Bates, 1888)

Heterosternus buprestoides Dupont, 1832

Heterosternus rodriguezi Candéze, 1869

Hexodon unicolor Olivier, 1789

Hologymnetis cinerea (Gory \& Percheron, 1833)

Hologymnetis undulata (Vigors, 1825)

Hoplia sp.

Hoplia chlorophana Erichson, 1847

Hoplia coerulea (Drury, 1773)

Hoplopyga liturata (Olivier, 1789) 
Inca clathrata (Olivier, 1792)

Ischnoscelis hoepfneri (Gory \& Percheron, 1833)

Isonychus sp.

Labarrus pseudolividus (Balthasar, 1941)

Lissomelas flohri Bates, 1889

Macraspis sp.

Macraspis aterrima (Waterhouse, 1881)

Macraspis rufonitida Burmeister, 1844

Macrodactylus sp.

Macrodactylus mexicanus Burmeister, 1845

Macrodactylus subspinosus Fabricius, 1775

Macrodactylus virens Bates, 1887

Macropoidelimus mniszechi (Sallé, 1873)

Macropoides crassipes (Horn, 1866)

Macropoides nietoi (Guérin-Méneville, 1844)

Marmarina maculosa (Olivier, 1789)

Mecynorhina polyphemus Fabricius, 1781

Megaceras sp.

Megasoma actaeon (Linnaeus, 1758)

Megasoma elephas (Fabricius, 1775)

Megasoma occidentalis Bolívar-Pieltain, Jiménez-Asua \& Martínez, 1963

Megasoma mars (Reiche, 1852)

Melolontha melolontha (Linnaeus, 1758)

Neoscelis dohrni (Westwood, 1855)

Oniticellus rhinocerulus Bates, 1889

Ontherus mexicanus Harold, 1868

Onthophagus sp.

Onthophagus alluvius Howden \& Cartwright, 1963

Onthophagus batesi Howden \& Cartwright, 1963

Onthophagus depressus Harold, 1871

Onthophagus coenobita (Herbst, 1783)

Onthophagus chevrolati Harold, 1869

Onthophagus crinitus Harold, 1869

Onthophagus furcatus (Fabricius, 1781)

Onthophagus hecate (Panzer, 1794)

Onthophagus hoepfneri Harold, 1869

Onthophagus igualensis Bates, 1887

Onthophagus incensus Say, 1835

Onthophagus lemur (Fabricius, 1781)

Onthophagus mexicanus Bates, 1887

Onthophagus nasicornis Harold, 1869

Onthophagus rhinolophus Harold, 1869

Onthophagus rostratus Harold, 1869

Onthophagus striatulus (Pasilot de Beauvois, 1809)

Onthophagus taurus (Schreber, 1759)

Onthophagus vacca (Linnaeus, 1767)

Oryctes boas (Fabricius, 1775) 
Oryctes nasicornis (Linnaeus, 1758)

Orizabus sp.

Orizabus isodonoides (Fairmaire, 1878)

Orizabus rubricollis (Prell, 1914)

Oxysternon conspicillatum (Weber, 1801)

Oxysternon lautum (MacLeay, 1819)

Oxythyrea funesta (Poda, 1761)

Pachnoda marginata (Drury, 1773)

Parachrysina parapatrica Deloya \& Morón, 1988

Parachrysina truquii (Thompson, 1857)

Paragymnetis flavomarginata poecila Schaum, 1848

Paratrichius doenitzi (Harols, 1879)

Pedinorrhina cinctuta (Voet, 1779)

Pelidnota sp.

Pelidnota aurescens Bates, 1888

Pelidnota frommeri Hardy, 1975

Pelidnota guatemalensisBates, 1888

Pelidnota nitescens Vigors, 1825

Pelidnota notata Blanchard, 1850

Pelidnota punctata (Linnaeua, 1758)

Pelidnota punctulata Bates, 1888

Pelidnota strigosa Laporte, 1840

Pelidnota virescens Burmeister, 1844

Pentodon algerinum (Herbst, 1789)

Phanaeus sp.

Phanaeus amithaon Harold, 1875

Phanaeus bispinus Bates, 1868

Phanaeus damocles Harold, 1863

Phanaeus daphnis Harold, 1863

Phanaeus demon Laporte, 1840

Phanaeus difformis LeConte, 1847

Phanaeus endymion Harold, 1863

Phanaeus florhi Nevinson, 1892

Phanaeus igneus MacLeay, 1819

Phanaeus melampus Harold, 1863

Phanaeus mexicanus Harold, 1863

Phanaeus obliquans Bates, 1889

Phanaeus palliatus Sturm, 1843

Phanaeus quadridens (Say, 1835)

Phanaeus vindex MacLeay, 1819

Phileurus didymus (Linnaeus, 1758)

Phileurus truncatus (Palisot de Beauvois, 1806)

Phileurus valgus (Linnaeus, 1758)

Phyllophaga sp.

Phyllophaga ravida (Blanchard, 1851)

Phyllopertha horticola (Fabricius, 1871)

Podischnus agenor (Olivier, 1789) 
Polyphylla sp.

Polyphylla petiti (Guérin-Méneville, 1844)

Popilia japonica Newman, 1838

Protaetia sp.

Protaetia affinis (Andersch, 1797)

Protaetia cuprea (Fabricius, 1775)

Protaetia morio (Fabricius, 1781)

Protaetia orientalis (Gory \& Percheron, 1833)

Pseudotorynorrhina japonica (Hope, 1841)

Ranzania splendens Berttoloni, 1855

Rutela lineola (Linnaeus, 1767)

Scarabeus sp.

Scarabeus laticollis Linnaeus, 1767

Spodistes mniszechi (Thompson, 1860)

Stenocrates ligneus Arrow, 1911

Stephanorrhina guttata (Olivier, 1789)

Strategus aloeus (Linnaeus, 1758)

Strategus antaeus (Drury, 1773)

Strategus longichomperus Ratcliffe, 1976

Strigoderma sp.

Strigoderma sulcipennis Burmeister, 1844

Sulcophanaeus chryseicollis Harold, 1863

Sisyphus schaefferi (Linnaeus, 1758)

Taurocerastes patagonicus Philippi, 1866

Tmesorrhina tridens Duvivier, 1891

Tomarus sp.

Tomarus bituberculatus (Palisot de Beauvois, 1811)

Tomarus laevicollis (Bates, 1888)

Tomarus nasatus (Burmeister, 1847)

Tomarus sallaei (Bates, 1888)

Trichius sp.

Trichius fasciatus (Linnaeus, 1758)

Trigonopeltastes delta (Forster, 1771)

Trigonophorus dilutus Bourgoin, 1914

Tropinota squalida (Scopoli, 1763)

Xyloryctes corniger Bates, 1888

Xyloryctes furcatus Burmeister, 1847

Xyloryctes howdenorum Delgado \& Nájera-Rincón, 1992

Xyloryctes telephus Burmeister, 1847

Xyloryctes thestalus Bates, 1888

Uroxys boneti Pereira \& Halffter, 1961

Buprestidae Leach, 1815

Lycidae Laporte, 1836

Lampyridae Latreille, 1817

Cantharidae Imhoff, 1856

Dermestidae Latreille, 1804

Trogossitidae Latreille, 1802 
Cleridae Latreille, 1802

Melyridae Leach, 1815

Nitidulidae Latreille, 1802

Erotylidae Latreille, 1802

Coccinellidae Latreille, 1807

Tenebrionidae Latreille, 1802

Oedemeridae, Latreille, 1810

Cerambycidae Latreille, 1802

Derobrachus sp.

Ochraethes sp.

Sphaenothecus luteicollis (Bates, 1885)

Stenaspis verticalis Serville, 1834

Stenygra histrio Serville, 1834

Trachyderes mandibularis (Dupont, 1834)

Tylosis puncticollis Bates, 1885

Chrysomelidae Latreille, 1802

Brentidae Billberg, 1820

Curculionidae Latreille, 1802 